\title{
Problema de dos cuerpos sometidos a varios tipos de fuerzas centrales
}

\author{
Luis Alcerro ${ }^{1}$ \\ ${ }^{1}$ Escuela de Física - UNAH, mail: luisalcerro@hotmail.com
}

Recibido: 20 de Febrero de 2016 / Aceptado: 01 de Mayo de 2016

\section{Resumen}

The two body problem in a central force is one of the most important models in classical mechanics because much physical systems are modeled in a similar way.

This paper develops this problem, obtaining the Kepler orbits, orbits caused by a force proportional to the inverse of distance's cube, and it ends with the orbits caused by a Yukawa's potential in the classical orbit equation.

Keywords: Orbit, Kepler, Yukawa.

El problema de dos cuerpos sometidos a una fuerza central es uno de los modelos mas importantes de la mecánica clásica debido a que muchos sistemas físicos se modelan de manera similar.

En el presente trabajo se desarrolla este problema, obteniendo las órbitas de Kepler, órbitas debido a una fuerza proporcional al inverso del cubo de la distancia, y se finaliza con la introducción de un potencial tipo Yukawa en la ecuación de órbita clásica.

Palabras clave: Órbita, Kepler, Yukawa

\section{LAGRANGIANO DEL SISTEMA}

Yrons ONSIDERE dos partículas de masas $m_{1}$ y $m_{2}$, ubicadas por los vectores $\vec{r}_{1}$ y $\vec{r}_{2}$ respectivamente.

Las únicas fuerzas en el sistema son $\vec{F}_{12}$ y $\vec{F}_{21}$ de su interacción mutua, las que asumimos conservativas y centrales, por tanto dichas fuerzas pueden deducirse de un potencial $U\left(\vec{r}_{1}, \vec{r}_{2}\right)$. Ya que el sistema es aislado

$$
U\left(\vec{r}_{1}, \vec{r}_{2}\right)=U\left(\vec{r}_{1}-\vec{r}_{2}\right)
$$

(traslacionalmente invariante.)

Ya que $\vec{F}_{12}$ y $\vec{F}_{21}$ son centrales

$$
U\left(\vec{r}_{1}-\vec{r}_{2}\right)=U\left(\left|\vec{r}_{1}-\vec{r}_{2}\right|\right)=U(r)
$$

El lagrangiano de este sistema es [6] :

$$
\mathcal{L}=\frac{1}{2} m_{1}(\dot{\vec{r}})^{2}+\frac{1}{2} m_{2}(\dot{\vec{r}})^{2}-U(r)
$$

\section{LAGRANGIANO RELATIVO Y DEL CENTRO DE MASA}

El vector de posicion del centro de masa se define:

$$
\dot{\vec{R}}=\frac{m_{1} \vec{r}_{1}+m_{2} \vec{r}_{2}}{m_{1}+m_{2}}=\frac{m_{1} \vec{r}_{1}+m_{2} \vec{r}_{2}}{M}
$$

Sabemos que el momento total de dos cuerpos es el mismo que el de una partícula con la masa total $\left(M=m_{1}+m_{2}\right)$ estuviera concentrada en el centro de masa:

$$
\vec{P}=M \dot{\vec{R}}
$$

De (2) se obtiene:

$$
\vec{r}_{1}=\vec{R}+\frac{m_{2}}{M} \vec{r} \quad, \quad \vec{r}_{2}=\vec{R}-\frac{m_{1}}{M} \vec{r}
$$

Entonces la energía cinética $T$ queda como:

$$
T=\frac{1}{2}\left[m_{1}(\dot{\vec{r}})^{2}+m_{2}(\dot{\vec{r}} 2)^{2}\right]=\frac{1}{2}\left[M(\dot{\vec{R}})^{2}+\frac{m_{1} m_{2}}{M}(\dot{\vec{r}})^{2}\right]
$$

haciendo $\mu=\frac{m_{1} m_{2}}{M}$ (masa reducida):

$$
T=\frac{1}{2} M(\dot{\vec{R}})^{2}+\frac{1}{2} \mu(\dot{\vec{r}})^{2}
$$

Así el lagrangiano en (1) queda como:

$$
\mathcal{L}=\mathcal{L}_{\mathrm{CM}}+\mathcal{L}_{\mathrm{Rel}}=\frac{1}{2} M(\dot{\vec{R}})^{2}+\left[\frac{1}{2} \mu(\dot{\vec{r}})^{2}-U(r)\right]
$$

\section{ECUACIONES DE MOVIMIENTO}

Recordando las ecuaciones de Euler-Lagrange:

$$
\frac{d}{d t} \frac{\partial \mathcal{L}}{\partial \dot{q}}=\frac{\partial \mathcal{L}}{\partial q}
$$

La ecuación de movimiento para $\vec{R}$, de 7 se obtiene:

$$
M \ddot{\vec{R}}=0 \Rightarrow \dot{\vec{R}}=\text { constante }
$$

Ésta última ecuación muestra la conservación del momento angular total.

La ecuación de movimiento para $\vec{r}$ :

$$
\mu \ddot{\vec{r}}=-\frac{d U}{d r}
$$


En coordenadas polares:

$$
(\dot{\vec{r}})^{2}=\dot{r}^{2}+r^{2} \dot{\phi}^{2}
$$

De (7) y 11) obtenemos:

$$
\mathcal{L}=\frac{1}{2} M(\dot{\vec{R}})^{2}+\frac{1}{2} \mu\left(\dot{r}^{2}+r^{2} \dot{\phi}^{2}\right)-U(r)
$$

Ya que $\dot{\vec{R}}=$ constante, se puede elegir como sistema de referencia inercial $\dot{\vec{R}}=0$. Entonces:

$$
\mathcal{L}=\frac{1}{2} \mu\left(\dot{r}^{2}+r^{2} \dot{\phi}^{2}\right)-U(r)
$$

Las ecuaciones de movimiento que se obtienen son:

$$
\begin{gathered}
\mu r^{2} \dot{\phi}=\text { constante }=l \\
\mu r \dot{\phi}^{2}-\frac{d U}{d r}=\mu \ddot{r}
\end{gathered}
$$

La ecuación 14 muestra la conservación del momento angular [1].

\section{ECUACIÓN DE LA ÓRBITA}

De (14) se obtiene que:

$$
\dot{\phi}=\frac{l}{\mu r^{2}}
$$

así (15) puede reescribirse

$$
\mu \ddot{r}=-\frac{d U}{d r}+\frac{l^{2}}{\mu r^{3}}
$$

donde, el segundo término de 17 es la fuerza centrífuga.

$$
F_{c f}=\frac{l^{2}}{\mu r^{3}}=-\frac{d}{d r}\left(\frac{l^{2}}{2 \mu r^{2}}\right)=-\frac{d U_{\mathrm{cf}}}{d r}
$$

con

$$
U_{\mathrm{cf}}=\frac{l^{2}}{2 \mu r^{2}} \text {. }
$$

Con las sustituciones anteriores, 17 se reescribe:

$$
\mu \ddot{r}=-\frac{d}{d r}\left[U(r)+U_{c f}\right]=-\frac{d}{d r} U_{e f f}(r)
$$

con

$$
U_{\mathrm{eff}}(r)=U(r)+U_{\mathrm{cf}}=U(r)+\frac{l^{2}}{2 \mu r^{2}}
$$

La ecuación radial en términos de la fuerza es:

$$
\mu \ddot{r}=F(r)+\frac{l^{2}}{\mu r^{3}} .
$$

Por regla de la cadena, podemos escribir:

$$
\frac{d}{d t}=\frac{d \phi}{d t} \frac{d}{d \phi}=\dot{\phi} \frac{d}{d \phi}=\frac{l}{\mu r^{2}} \frac{d}{d \phi}
$$

y con la sustitución:

$$
u=\frac{1}{r} \quad, \quad r=\frac{1}{u},
$$

$$
\frac{d}{d t}=\frac{l u^{2}}{\mu} \frac{d}{d \phi}
$$

entonces:

$$
\begin{gathered}
\dot{r}=\frac{d r}{d t}=\frac{l u^{2}}{\mu} \frac{d r}{d \phi}=\frac{l u^{2}}{\mu} \frac{d}{d \phi}\left(\frac{1}{u}\right)=-\frac{l}{\mu} \frac{d u}{d \phi} \\
\ddot{r}=\frac{\dot{r}}{d t}=-\frac{l^{2} u^{2}}{\mu^{2}} \frac{d^{2} u}{d \phi^{2}}
\end{gathered}
$$

Sustituyendo (24) y 27 en $(22)$ se obtiene la ecuacion de la órbirta[2]:

$$
u^{\prime \prime}(\phi)=-u(\phi)-\frac{\mu}{l^{2} u(\phi)^{2}} F
$$

\section{V. Órbitas de Kepler}

Para encontrar las orbitas de Kepler, se debe introducir la fuerza gravitacional

$$
F(r)=-\frac{\gamma}{r^{2}}=-\gamma u^{2} \quad, \quad \gamma=G m_{1} m_{2}
$$

en 28):

$$
u^{\prime \prime}(\phi)=-u(\phi)+\frac{\gamma \mu}{l^{2}}
$$

Haciendo la sustitución:

$$
w(\phi)=u(\phi)-\frac{\gamma \mu}{l^{2}}
$$

se obtiene

$$
w(\phi)=A \cos (\phi-\delta) \Rightarrow u(\phi)=\frac{\gamma \mu}{l^{2}}+A \cos (\phi-\delta)
$$

Convenientemente se pude tomar $\delta=0$ :

$$
u(\phi)=\frac{\gamma \mu}{l^{2}}+A \cos (\phi)=\frac{\gamma \mu}{l^{2}}\left(1+\frac{A l^{2}}{\gamma \mu} \cos (\phi)\right)
$$

Haciendo

$$
\epsilon=\frac{A l^{2}}{\gamma \mu}
$$

entonces

$$
u(\phi)=\frac{\gamma \mu}{l^{2}}(1+\epsilon \cos (\phi))
$$

Realizando otra sustitución:

$$
c=\frac{l^{2}}{\gamma \mu}
$$

se obtiene:

$$
r(\phi)=\frac{c}{1+\epsilon \cos \phi}
$$

la cual es la trayectoria de la órbita [6].

El tipo de órbita dependerá de la excentricidad $(\epsilon)$ :

\begin{tabular}{|c|c|}
\hline Excentricidad & Órbita \\
\hline$\epsilon=0$ & Círculo \\
\hline $0<\epsilon<1$ & Elipse \\
\hline$\epsilon=1$ & Parábola \\
\hline$\epsilon>1$ & Hipérbola \\
\hline
\end{tabular}




\section{Gráficas de Órbitas de KePler}

Procedemos a graficar las órbitas de Kepler. Usando (37) y tomando $c=1$, la ecuación de la órbita queda totalmente determinada por la excentricidad $(\epsilon)$.

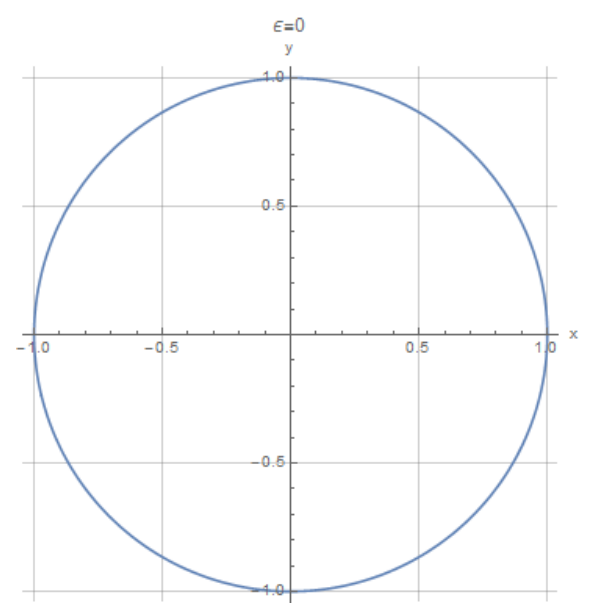

Figura 1: Órbita circular

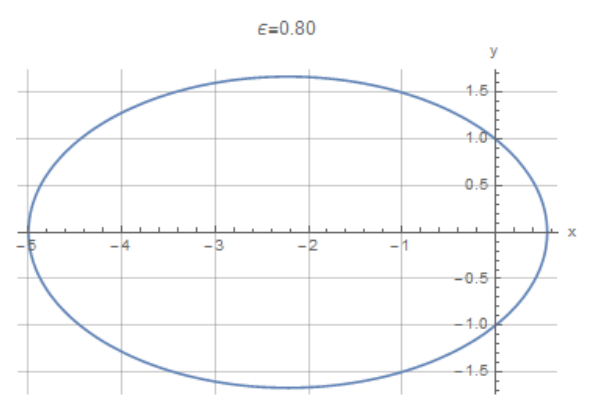

Figura 2: Órbita elíptica

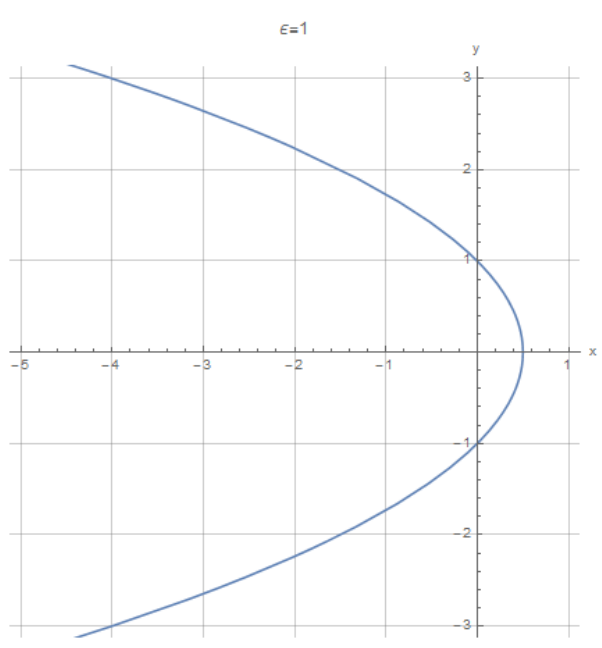

Figura 3: Órbita parabólica

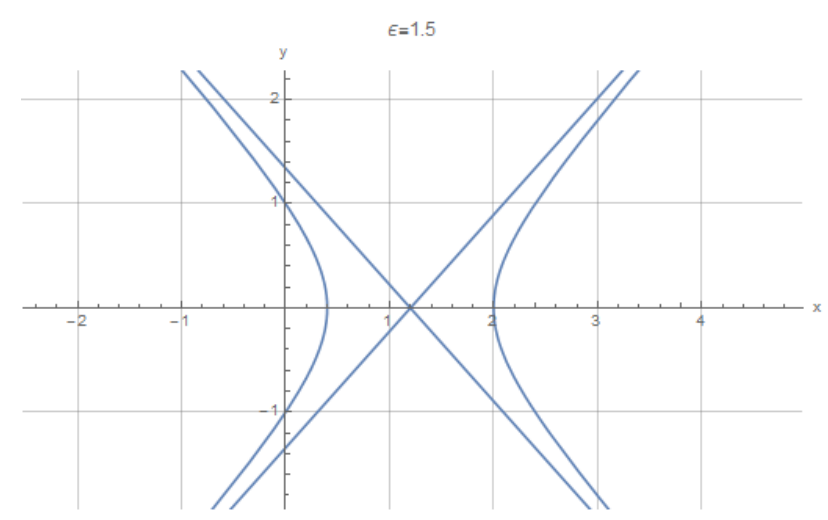

Figura 4: Órbita hiperbólica

VII. ÓRBITAS CON UNA FUERZA TIPO

$$
F=-\gamma / r^{3}
$$

Recordando:

$$
u^{\prime \prime}(\phi)=-u(\phi)-\frac{\mu}{l^{2} u(\phi)^{2}} F
$$

Si:

$$
F=-\frac{\gamma}{r^{n}}
$$

entonces

$$
u^{\prime \prime}(\phi)=-u(\phi)+\frac{\gamma \mu}{l^{2}} u^{n-2}(\phi)
$$

Si $n=2 \Rightarrow$ Órbitas de Kepler.

Si $n=3$ obtenemos la ecuación

$$
u^{\prime \prime}(\phi)+k u(\phi)=0
$$

donde

$$
k=1-\frac{\gamma \mu}{l^{2}}
$$

Si $k=0 \Rightarrow \frac{\gamma \mu}{l^{2}}=1$. Entonces

$$
u^{\prime \prime}(\phi)=0
$$

y esta tiene como solución:

$$
u(\phi)=C_{1}+C_{2} \phi \Rightarrow r(\phi)=\frac{1}{C_{1}+C_{2} \phi}
$$

La trayectoria de esta órbita se muestra a continuación:

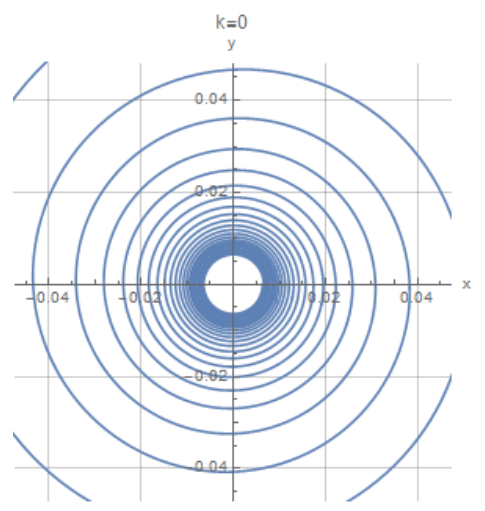

Figura 5: Órbita con $k=0, C_{1}=1, C_{2}=1$ 
Si $k>0$ la solución a 41) es:

$$
r(\phi)=\frac{1}{C_{1}+C_{2} \exp (-k \phi)}
$$

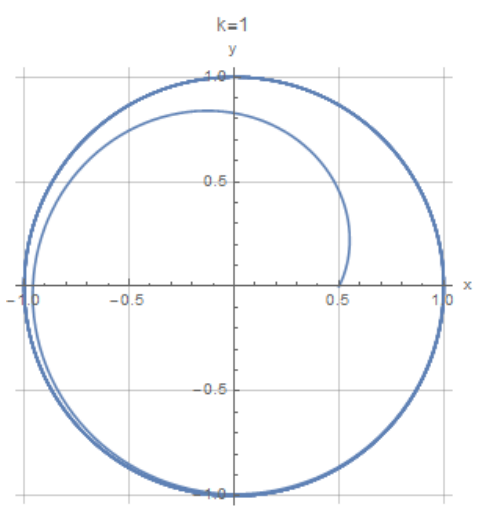

Figura 6: Órbita con $k=1, C_{1}=1, C_{2}=1$

Si $k<0$ la solución a (41) es:

$$
r(\phi)=\frac{1}{C_{1}+C_{2} \exp (-k \phi)}
$$

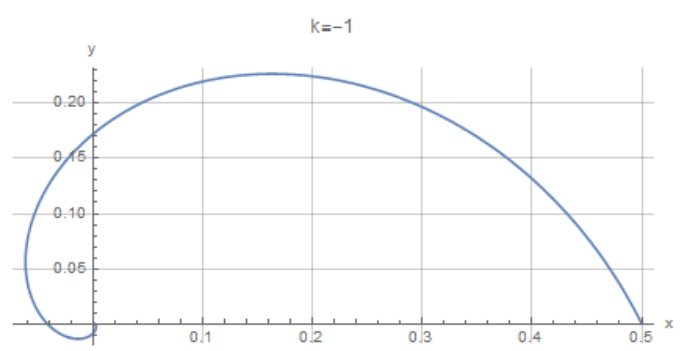

Figura 7: Órbita con $k=-1, C_{1}=1, C_{2}=1$

\section{VIII. ÓRBITAS EN UN POTENCIAL TIPO YUKAWA}

En 1934, el físico Hideki Yukawa propuso un modelo para la interacción nuclear fuerte [3]. El conjeturó un potencial que mantenía unido al núcleo.

Analizarémos las posibles órbitas obtenidas con el potenciál de Yukawa en la ecuación de orbita clásica.

Introducimos el potencial de Yukawa como la interacción entre dos nucleones a una distancia $r$, el cual tiene la forma [5]:

$$
U(r)=\frac{-k}{r} e^{\frac{-r}{a}}
$$

con $k>0, a>0$.

Analizaremos primeramente las condiciones para que la órbita sea abierta o cerrada.

De (21), tenemos que nuestro potencial efectivo tomaría la forma:

$$
U_{\text {eff }}=\frac{-k}{r} e^{\frac{-r}{a}}+\frac{l^{2}}{2 \mu r^{2}}
$$

de modo que podemos definir el potencial adimensional:

$$
w(x)=\frac{a}{k} U_{\text {eff }}=\frac{e^{-x}}{x}+\frac{\beta}{2 x^{2}}
$$

con $x=r / a$ y $\beta=l^{2} / a k \mu$.

De la misma forma, definimos:

$$
\epsilon=\frac{a E}{k}
$$

Así, debemos encontrar las raíces de la ecuación

$$
w(x)=\epsilon
$$

para determinar si existen puntos de retorno.

Determinando los extremos de $w(x)$, resolvemos la ecuación

$$
w^{\prime}(x)=0
$$

o de forma equivalente,

$$
x(x+1) e^{-x}=\beta
$$

Nos interesa saber si esta ecuación tiene raíces o no, por tanto derivamos la parte derecha y la igualamos a cero, obteniendo:

$$
x_{o}=\frac{1+\sqrt{5}}{2}
$$

así, el lado derecho de (53) en éste punto es:

$$
y_{o}=\left(\frac{1+\sqrt{5}}{2}\right)\left(\frac{2+\sqrt{5}}{2}\right) \exp \left(\frac{1+\sqrt{5}}{2}\right) \approx 0.84
$$

Por tanto, si $\beta>y_{o}$ la órbita es abierta. Caso contrario, $\beta<y_{o}$, la órbita será cerrada.

Consideremos el caso en que $k=l=\mu=1$, y hagamos $\beta=y_{o}$ para determinar las condiciones en la constante $a$,

$$
\beta=\frac{l^{2}}{a k \mu}=\frac{1}{a}=y_{o} \Rightarrow a \approx 1.19
$$

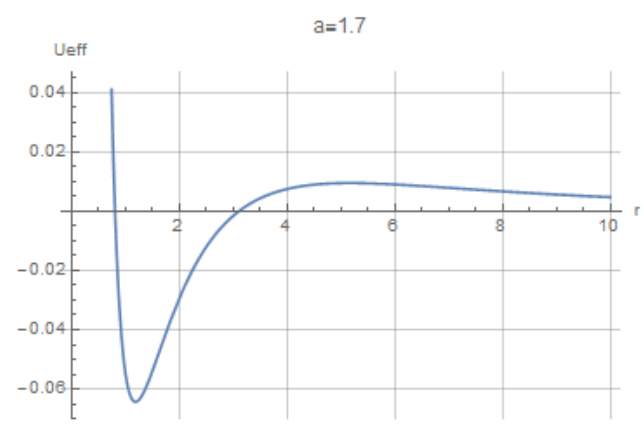

Figura 8: Potencial efectivo para $a=1.7$ 


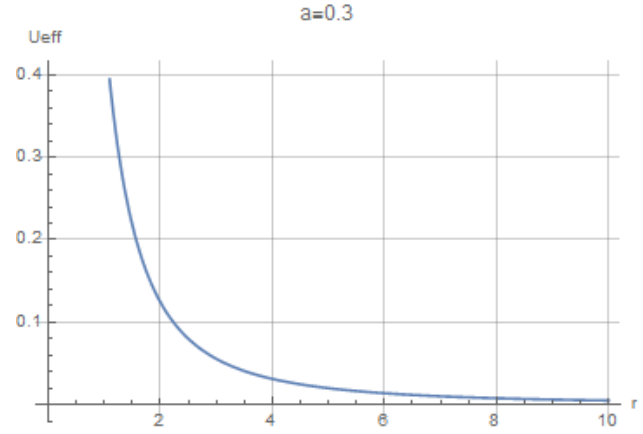

Figura 9: Potencial efectivo para $a=0.3$

Sabiendo las condiciones anteriores, podemos proceder a encontrar las órbitas que sigue una partícula sometida a éste potencial.

De (47), tenemos:

$$
F(r)=\frac{d U(r)}{d r}=-\left(\frac{k}{r^{2}} e^{\frac{-r}{a}}+\frac{k}{a r} e^{\frac{-r}{a}}\right)
$$

de modo que

$$
F(u)=-\left(k u^{2} e^{\frac{-1}{a u}}+\frac{k}{a} u e^{\frac{-1}{a u}}\right)
$$

Introduciendo ahora 58 en 28 , se obtiene:

$$
\begin{aligned}
u^{\prime \prime}(\phi) & =-u(\phi)+ \\
& +\frac{\mu}{l^{2} u(\phi)^{2}}\left(k u(\phi)^{2} e^{\frac{-1}{a u(\phi)}}+\frac{k}{a} u(\phi) e^{\frac{-1}{a u(\phi)}}\right)
\end{aligned}
$$

Resolveremos la ecuación diferencial anterior de forma numérica, con los valores elegidos anteriormente para $a \mathrm{y}$ $k$.

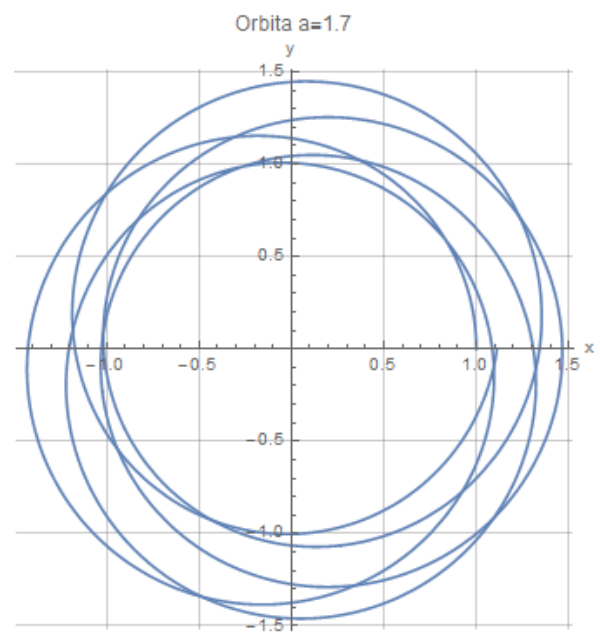

Figura 10: Órbita obtenida para un valor de $a=1.7$ y $k=1$

Con la gráfica de la figura 10, no podríamos determinar si en efecto se trata de una órbita cerrada. Sin embargo si ampliamos el rango de la solución, esperamos que esta solución esté acotada ya que $\mathrm{a}=1.7$.

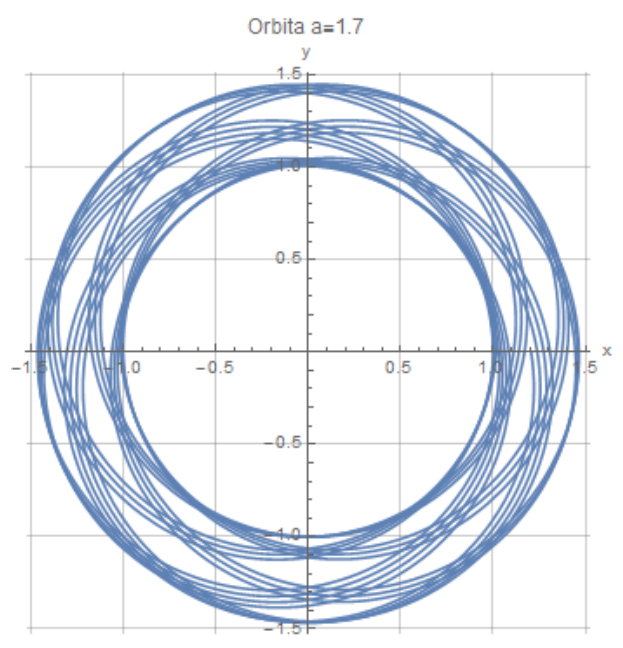

Figura 11: Órbita obtenida para un valor de $a=1.7$ y $k=1$

Como ejemplo de una órbita abierta, tomamos $\mathrm{a}=0.3$.

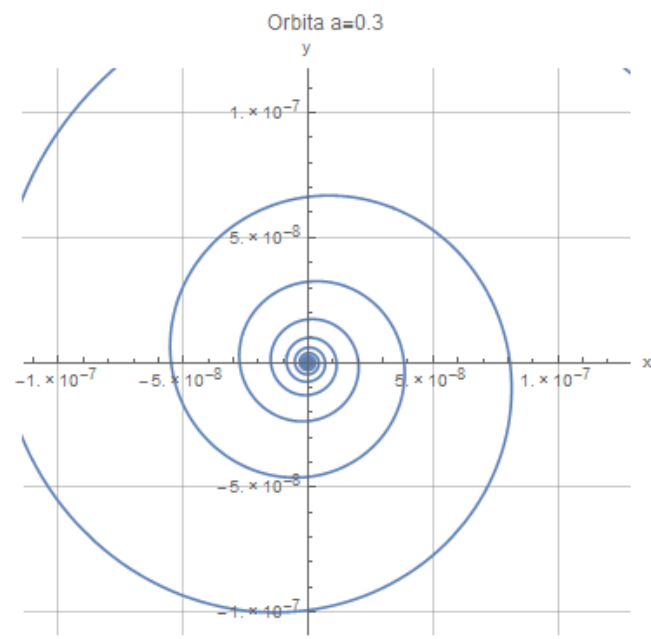

Figura 12: Órbita obtenida para un valor de $a=0.3$ y $k=1$

\section{Conclusiones}

- La ecuación de órbita clásica nos permite introducir cualquier potencial con la única restricción que este sea central y así poder observar las distintas órbitas que se obtienen.

- Al introducir potenciales a la ecuación de la órbita, obtenemos una ecuación diferencial que, por le general, es no lineal. Debido a esto nos vemos obligados a acudir a las herramientas numéricas computacionales para tratar este tipo de problemas.

- La estabilidad de una órbita depende de las condiciones iniciales, es decir de la energía mecánica de esta [4]. 


\section{REFERENCIAS}

[1] Goldstein, H.; Poole, C. y Safko, J. (2001). Classical Mechanics. Pearson Education Limited.

[2] Gregory, R. (2006). Classical Mechanics. Cambridge University Press.

[3] Griffiths, D. (2008). Introduction to Elementary Particles. Physics textbook. Wiley.
[4] Kibble, T. y Berkshire, F. (2004). Classical Mechanics. Imperial College Press.

[5] Ryder, L. (1996). Quantum Field Theory. Cambridge University Press.

[6] TaYlor, J. (2005). Classical Mechanics. University Science Books. 